ISSN: 0213-2060

DOI: https://doi.org/10.14201/shhme2020382221238

\title{
LOS PACIENTES DEL HOSPITAL DE SAN JUAN EN EL REINO LATINO DE JERUSALÉN
}

\author{
The Patients of the Hospital of St John in the Latin Kingdom of Jerusalem
}

\author{
Esteban GREIF \\ Instituto Multidisciplinario de Historia y Ciencias Humanas-CONICET. Facultad de Filosofia y Letras. Universidad \\ de Buenos Aires. Fátima 3508, Castelar, CP 1712 BUENOS AIRES. Argentina. C. e.: estebangreif1184@gmail.com
}

Recibido: 2020-01-28

Revisado: 2020-04-18

Aceptado: $2020-10-26$

RESUMEN: La empresa de construcción y puesta en marcha del hospital de San Juan de Jerusalén expresaba la enorme capacidad de concentración de recursos, así como el protagonismo en la atención médica que poseían los hermanos hospitalarios dentro del Reino Latino de Jerusalén. Sin embargo, y pese a su importancia, se ha discutido el grado de desarrollo médico que la institución efectivamente alcanzó y si los servicios que ofrecía tendían al simple cuidado y hospedaje de personas convalecientes o a la curación de pacientes enfermos. En este trabajo proponemos una relectura de un conjunto diverso de documentos que describen la tarea desplegada por la orden para, de esta manera, lograr una mejor comprensión del carácter específico de esta institución. médicos.

Palabras clave: Hospitalarios; Hospital en Jerusalén; Desarrollo; Pacientes; Servicios

ABSTRACT: The construction and operation of the Hospital of St John expressed the enormous capacity for the concentration of resources and the leadership in medical attention that the Hospitallers hold in the Latin Kingdom of Jerusalem. However, and despite its importance, it has been discussed the medical degree that the institution really achieved and if the services offered within were heading to the simple care and lodging of convalescent people or to the cure of sick patients. In this paper we propose a reassessment of a set of different documents that described the work deployed by the order, to get a better comprehension of the specific character of this institution.

Keywords: Hospitallers; Hospital in Jerusalem; Development; Patients; Medical services. 
SUMARIO: 1 Acerca de los pacientes del Hospital. 2 Los pacientes del Hospital ¿pobres o enfermos? 3 Los servicios médicos del Hospital. 4 El cuidado a los niños. 5 Conclusiones. 6 Referencias bibliográficas.

\section{Acerca de los pacientes del Hospital ${ }^{1}$}

A la hora de estudiar las instituciones hospitalarias de la Edad Media predominaron en la historiografía dos posiciones, a saber, la de los historiadores "optimistas» ${ }^{2}$ y la de los «pesimistas». La divergencia en los enfoques sobre la evolución institucional de la medicina ha sido en gran medida producto de dichas interpretaciones. De este modo, como afirmó Peregrine Horden, la discusión giró en torno al grado de «medicalización» alcanzado por los hospitales medievales. Es decir, la definición y el entendimiento de las instituciones hospitalarias a partir de la presencia regular de médicos designados con el propósito de curar a las personas que residían en ellas. En este sentido, mientras que para los «pesimistas» sería imposible registrar el origen de las instituciones hospitalarias actuales en el mundo medieval, para los "optimistas» los hospitales modernos serían, en mayor o menor medida, herederos de los que existieron en el Mediterráneo oriental desde el siglo IV $^{3}$.

En sus comienzos, estas instituciones eran hospicios dentro de los monasterios bizantinos que recibían pobres, peregrinos, extranjeros y huérfanos que quedaban bajo el cuidado de sus monjes ${ }^{4}$. En los siglos siguientes, fueron fundadas muchas de estas instituciones en Constantinopla y en ciudades de envergadura como Alejandría, Antioquía o Cesarea, siguiendo patrones similares a las originales 5 . Llamado nosokomeion o xenodochion, el hospital comenzó a emplear el personal adecuado para la curación de los enfermos. En este sentido, el siglo vi conoció una evolución notable con la decisión de Justiniano de desafectar de los dispensarios municipales a los archiatroi-médicos locales

1 Designaremos al hospital en Jerusalén con minúscula, para distinguirlo del Hospital con mayúscula cuando nos refiramos a los caballeros hospitalarios y no a la institución hospitalaria.

2 El autor más representativo de esta postura es Timothy Miller. En este sentido, sostuvo que el hospital bizantino fue el modelo sobre el que se desarrollaron los hospitales occidentales. Miller, Timothy. The birth of the hospital in the Byzantine Empire. Baltimore-London: The John Hopkins University Press, 1997. Cfr. Horden, Peregrine. "The Earliest Hospitals in Byzantium, Western Europe, and Islam». The Journal of Interdisciplinary History, 2005, vol. 35, n. ${ }^{\text {3 }}$, pp. 388-389.

3 Horden, Peregrine. «How Medicalised Were Byzantine Hospitals?». Medicina \& Storia, 2006, vol. 5, n. ${ }^{\circ} 10$, pp. $45-74$.

4 Sobre el aspecto filantrópico del mundo bizantino véase la obra de Demetrios Constantelos. ConsTANTElos, Demetrios J. Byzantine philanthropy and social welfare. New Brunswick-New Jersey: Rutgers University Press, 1968. Acerca de la asociación de la atención hospitalaria a la caridad cristiana bizantina véase, entre otros, Allan, Nigel. "Hospice to Hospital in the Near East: An Instance of Continuity and Change in Late Antiquity». Bulletin of the History of Medicine, 1990, vol. 64, n.o 3, pp. 446-462. CongourdeAu, Marie-Hélène. "La médecine byzantine. Une réévaluation nécessaire». Revue du Praticien, 2004, vol. 54, n. ${ }^{\circ}$ 15, pp. 1733-1737.

5 Miller, Timothy. «Byzantine hospitals». Dumbarton Oaks Papers, 1984, vol. 38, pp. 53-63. 
de reconocida competencia bajo el servicio del estado- y asignarlos, en cambio, a los hospitales monásticos bajo la dirección del obispo responsable de estas instituciones ${ }^{6}$.

Además de los médicos, los hospitales bizantinos contaban entre los miembros de su personal con asistentes encargados de la aplicación de medicinas, el registro del progreso médico de los pacientes e, incluso, la realización de operaciones menores. Para el siglo XII los hospitales bizantinos empleaban también personal de limpieza, cocineros, farmacéuticos y de cuidado del material quirúrgico ${ }^{7}$.

Por otro lado, se ha asumido que en el mundo islámico medieval el surgimiento de los hospitales - Bìmāristans - tuvo lugar hacia el siglo vin en Bagdad, cuando el califa abásida al-Rashid fundó la primera de estas instituciones alrededor del año 790 en la capital de su califato. La expansión posterior de dicha institución fue lenta, pero ya hacia el siglo XII la mayoría de las ciudades bajo el Islam poseían al menos un hospital, mientras que las mayores, como Bagdad, varios de ellos. Cabe destacar que los hospitales del Islam fueron herederos en gran medida de aquellos del mundo bizantino, así como sus profesionales de los conocimientos médicos de sus pares del Oriente Cristiano ${ }^{8}$.

Por último, en el mundo europeo medieval la palabra para designar qué era un hospital (hospitale) se refería a un significado bastante amplio de instituciones, a diferencia de su sentido más estricto en el Cercano Oriente. De tal modo, a veces se designaba bajo el mismo término a los hospicios para pobres como a las instituciones que albergaban a aquellos que sufrían una condición incurable, como los leprosos (leprosaria). Otros hospitalia funcionaban como casas de retiro para los frailes cuando envejecían (hospicium). Por otro lado, mientras algunas de estas instituciones daban habitación y alimento a peregrinos y viajeros, al menos hasta fines del siglo XII, solo un pequeño número de ellas se dedicaban a la atención y curación del enfermo?

Las instituciones donde tuvo lugar un desarrollo médico más significativo en la atención y cuidado del enfermo durante la Alta Edad Media europea fueron fundamentalmente las enfermerías de los monasterios. Dicha atención se dirigía principalmente a los hermanos del convento monacal y, en segundo lugar, a los enfermos pobres de la zona $^{10}$. Sin embargo, conviene seńalar que el desarrollo material y organizativo de estas instituciones no era de la dimensión de aquellas del mundo oriental, situación que comenzaría a modificarse desde finales del siglo XII. Desde entonces, en el Occidente me-

6 Congourdeau, «La médecine byzantine», pp. 1734-1735.

7 Una vision de conjunto sobre los hospitales bizantinos, su evolución y funcionamiento en MiLLER, The birth of the hospital in the Byzantine Empire.

8 Sobre el origen, funcionamiento y características de los hospitales y la medicina del Islam medieval véase Hamarneh, Sami. "Development of hospitals in Islam». Journal of the History of Medicine and Allied Sciences, 1962, vol. 17, pp. 366-384. Cfr. Dols, Michael. "The origins of the Islamic hospital: myth and reality». The Bulletin of the History of Medicine, 1987, vol. 61, pp. 367-390. De publicación reciente véase Ragab, Ahmed. The Medieval Islamic Hospital. Medicine, Religion and Charity. New York: Cambridge University Press, 2015. Ragab incorpora las tesis sobre un desarrollo posterior del hospital en el mundo islámico y los mitos de origen del primer hospital en Bagdad.

9 Risse, Gunter. Mending Bodies, saving souls: A history of hospitals. New York: Oxford University Press, 1999, pp. 69-165.

10 Davis, Adam J. The Medieval Economy of Salvation. Charity, Commerce, and the Rise of the Hospital. Ithaca: Cornell University Press, 2019. 
dieval comenzaron a surgir algunos hospitales que se dedicaron a la atención de aquellos que sufrían enfermedades o lesiones, junto a las restantes tareas que allí se desplegaban, como las de asilo a pobres y peregrinos ${ }^{11}$.

Retomando la discusión sobre el grado de medicalización de las instituciones hospitalarias medievales, el punto interesante en torno a nuestro trabajo reside en su vínculo con lo postulado por algunos historiadores de la orden del Hospital sobre los sujetos que eran atendidos en su domus Dei. La historia de los hospitalarios comenzó en el año 1071, cuando un grupo de mercaderes amalfitanos reconstruyó el monasterio benedictino de Santa María de los Latinos junto al hospicio asociado en Jerusalén ${ }^{12}$. Luego de ser renovado, los amalfitanos emplearon para la dirección del complejo a un grupo de hermanos benedictinos que a cambio de la reconstrucción les otorgarían atención y hospedaje a los mercaderes de dicha ciudad que llegaban a Tierra Santa. Al mismo tiempo, también se fundó un convento de monjas para la atención de las mujeres en el hospicio de Santa María Magdalena, cuya primera abadesa se llamó Agnes ${ }^{13}$.

Luego de la Primera Cruzada los mercaderes amalfitanos decidieron crear un tercer hospicio y otra iglesia para la atención de todos los viajeros pobres sin importar su origen. La hermandad a cargo se conoció con el nombre de los hospitalarios. En pocos años estos hermanos organizaron un eficiente servicio de atención hospitalaria e iniciaron un proceso de transformación que los llevaría a convertirse en una de las órdenes religiosas más poderosas del Oriente latino y del mundo occidental ${ }^{14}$. Para mediados de la década del '50 del siglo xir los hospitalarios habían terminado la construcción del gran hospital que reemplazó a los hospicios sostenidos por la orden desde su origen hasta la caída de la ciudad bajo las tropas de Saladino en el año $1187^{15}$.

Algunos historiadores se preguntaron si esta institución era «legítimamente» considerable como un hospital. Es decir, si desplegaba un elevado grado de desarrollo médico para los pacientes que atendía. Algunas preguntas giraban en torno a cómo considerar a los enfermos residentes en el hospital ¿eran peregrinos que necesitaban hospedaje y comida para continuar su viaje?, o, más bien, ¿eran pobres en búsqueda de un refugio?;

11 Brodman, James W. «Religion and Discipline in the Hospitals of Thirtennth-century France». En Bowers, Barbara S. (ed.). The Medieval Hospital and Medical Practice. London-New York: Routledge, 2007, pp. 123-132.

12 Demurger, Alain. Les Hospitaliers. De Jerusalem à Rhodes. 1050-1317. Paris: Éditions Tallandier, 2015, pp. 54-57.

${ }_{13}$ Huygens, Robert (ed.). Guillaume du Tyr. Chronicon, Corpus Christianorum. Continuatio Medievalis, vols. 63, 63A. Turnhout: Brepols Publishers, 1986, pp. 816-817.

${ }^{14}$ Para la cronología de la fundación y su vínculo con Amalfi véase Riley-Smith, Jonathan. The Knights Hospitallers in the Levant, c. 1070-1309. Hampshire: Palgrave Macmillan, 2012, pp. 15-18. Para una lectura general sobre el tema específico de las actividades desplegadas por la orden para el servicio de los peregrinos y pobres en el Oriente Latino desde sus orígenes hasta la expulsión de los cruzados de Outremer, véase Bronstein, Judith. «Servus pauperum Christi: los servicios de los Hospitalarios a los peregrinos en el Oriente Latino, siglos XI-XIII». Memoria y Civilización, 2013, vol. 16, pp. 219-236.

15 Boas, Adrian. Archeology of the Military Orders. London: Routledge, 2006, p. 44. Sobre la evolución de dicho complejo, así como su surgimiento y la existencia de iglesias y edificios conventuales véase PRINGLE, Denys. The Churches of the Crusader Kingdom of Jerusalem; A Corpus 3: The City of Jerusalem. Cambridge: Cambridge University Press, 1993, pp. 193-207. 
si eran pobres, ¿lo eran por su condición socioeconómica, o por su estado de salud?; y, si eran enfermos con determinadas dolencias, ¿qué terapias brindaba el Hospital? ${ }^{16}$

En torno a esta cuestión Susan Edgington señalaba que para 1140 las fuentes del Hospital se referían a sus pacientes bajo el término infirmi. Al mismo tiempo, la autora indicaba que, equivocadamente, en reiteradas ocasiones dicho término fue traducido como «enfermo» en el sentido estricto con el que hoy lo entenderíamos. Aclaraba, además, que la palabra correcta para esta designación correspondía al vocablo latino de aegri, cuyo registro en las fuentes, afirmaba la autora, es prácticamente nulo.

Según sostenía la historiadora inglesa, solo una vez en los documentos del Hospital previos al año 1187 aparecía la mención de los beneficiarios de la tarea de la orden como egroti $i^{17}$. Para ella, en efecto, el propósito del Hospital, incluso en el año 1180, era hospedar a los pobres y a los peregrinos que sufrían de malnutrición y agotamiento, y, quizás, el cuidado de aquellos que padecían algún tipo de condición crónica.

Por lo tanto, para Edgington la orden en Jerusalén solo proveyó un sistema de atención orientado al cuidado de sus residentes, antes que a su curación. En este sentido, agregaba que la analogía moderna con este sistema de atención no sería el hospital, sino el hogar para los convalecientes. Asimismo, sostenía que el término más común para designar a los residentes en las fuentes de la institución de Jerusalén era el de pauperes $^{18}$.

En un sentido opuesto, Benjamin Kedar advirtió que ya el hospital creado por los amalfitanos podría haber funcionado no solo como hospicio para pobres y peregrinos, sino como una institución encargada de la curación de los enfermos. Contrario a Edgington, el autor consideraba que incluso el primer hospicio de la orden antes de la construcción del gran palacio probablemente contratara médicos para la provisión de atención médica profesional. Sin embargo, esta última afirmación sería una presunción que el autor no podría comprobar con seguridad ${ }^{19}$.

En función de estas afirmaciones decidimos, en primer lugar, tratar de dilucidar algunos aspectos con relación al uso de los diferentes términos que designaban a los

16 De la misma manera podríamos preguntarnos sobre la dificultad de establecer distinciones tajantes ente asistencia y tratamiento médico sin caer en divisiones que resultan anacrónicas para el período estudiado. En este sentido, para una aproximación general a la discusión sobre el carácter religioso del hospital medieval, véase Brodman, James. Charity and Welfare: Hospitals and the Poor in Medieval Catalonia. Philadelphia: University of Pennsylvania Press, 1998. De reciente publicación, véase también, Davis, The Medieval Economy of Salvation.

17 Delaville le Roulx, Joseph (ed.). Cartulaire général de l’Ordre des Hospitaliers de S. Jean de Jerusalem, 4 vols. Paris: Académie Royale des Inscriptions et Belles-Lettres, 1895-1906, n. ${ }^{\circ}$ 471, pp. 323-324 (en adelante se citará el número del Cartulario con el número de página).

18 "In short, in normal times the overwhelming concern of the hospital was not curing but caring, which within the frame of contemporary scientific knowledge meant an emphasis on spiritual and physical comfort, and on dietary regulation in accordance with humoral theory. The modern analogy is not the hospital, but the convalescent home». Edgington, Susan. "The Hospital of St John in Jerusalem». En Amar, Zohar; Lev, Efrain y Schwartz, Joshua (eds.). Medicine in Jerusalem throughout the ages. Tel Aviv: Eretz, 1999, pp. XXI-XXII.

${ }_{19}$ Kedar, Benajmin. "A note on Jerusalem's Bimaristan and Jerusalem's Hospital». En Borchard, Karl; Jaspert, Nikolas y Nicholson, Helen (eds.). The Hospitallers, the Mediterranean and Europe Festschrift for Anthony Luttrell. London: Ashgate, 2007, pp. 7-11. 
residentes del hospital para, de esta forma, aclarar el significado de dichos términos. Con relación a esto, en segundo lugar, registrar si efectivamente el término aegri (o las flexiones correspondientes) no figura, como señaló Susan Edgington, en las fuentes correspondientes a los años previos a 1180 que figuran fundamentalmente en los cartularios de la orden de San Juan en Jerusalén. En tercer lugar, ampliar nuestro registro sobre esta palabra hacia otras fuentes que las consultadas por esta autora y ver si contienen referencias sobre la condición de los pacientes del Hospital. En este sentido, nos detendremos no solo en el análisis de las fuentes regulares de los hospitalarios, sino en las descripciones realizadas por diversos peregrinos que residieron u observaron la tarea médica desplegada por los hermanos del Hospital, como el Testimonio Anónimo realizado por un peregrino que residió en la domus Dei en la Ciudad Santa hacia finales del siglo XII y elaboró la descripción más rica sobre la vida y la atención dispensada a los pobres y los enfermos en el hospital de Jerusalén ${ }^{20}$. Con relación a este punto, por último, decidimos profundizar sobre las características de los sujetos que eran recibidos en el Hospital y los servicios que dispensaban los hermanos hospitalarios y así obtener una dimensión más adecuada acerca de la tarea médica que desplegaron en Jerusalén.

\section{Los pacientes del Hospital ¿POBRES O ENFERMOS?}

En torno al primer punto señalado, un problema central deriva de lo que entendemos por «pobres» $\mathrm{y}$ «enfermos» en relación con la atención hospitalaria medieval. Sobre este aspecto, conviene aclarar que mediante estas categorías muchas veces se designaba a los mismos sujetos. Es decir, pauperes servía para identificar a los pobres (estructurales u ocasionales) que podían sufrir, o no, una enfermedad. Pero también, se refería a los enfermos que, como afirmaba Alain Demurger, «(...) no son necesariamente miserables, pero que, porque están enfermos, devienen en pobres de Cristo. Situación económica y rango social, situación sanitaria y situación espiritual están entonces inextricablemente mezclados $\aleph^{21}$. Por lo tanto, consideramos que resulta problemático definir las tareas que el hospital desplegaba y su grado de medicalización en función de la aparición de estos

20 El texto latino del Clérigo Anónimo forma parte del manuscrito Munich Stadts bibliothek Clm 4620 del que ocupa los folios 132v a 139v. Dicho manuscrito está datado en el siglo XIII y es la única copia que se conserva de ese texto, a partir de la cual existen dos ediciones. La primera fue realizada por Benjamin Kedar en 1998, mientras que la segunda -acompańada de una traducción al francés- lo fue por Alain Beltjens en 2004. Respectivamente, Kedar, Benjamin. "A twelfth-century description of the Jerusalem Hospital». En Nicholson, Helen (ed.). The Military Orders: fighting for the faith and caring for the sick. Vol. 2. London: Ashgate, 1998; Beltjens, Alain. "Le récit d'une journée au Grand Hôpital de Saint-Jean de Jérusalem sous le règne des derniers rois latins ayant résidé à Jérusalem ou le témoignage d'un clerc anonyme conservé dans le manuscrit Clm. 4620 de Munich». Société de l'Histoire et du Patrimoine de l'Ordre de Malte. Numéro spécial, 2004, vol. 14, pp. 1-79. Nos basaremos aquí en esta última edición (en adelante se citará como Clérigo Anónimo y el número de página).

21 «(...) ne sont pas nécessairement des miséreux, mais qui, parce qu’ils sont malades, deviennent des pauvres du Christ. Situation économique et rang social, situation sanitaire et situation spirituelle sont donc inextricablement mêlées». Demurger, Les Hospitaliers, p 20. 
términos en las fuentes debido a su polisemia y la dificultad de precisar su sentido dentro de las categorías actuales.

En efecto, si analizamos algunas de las fuentes del Hospital, muchas veces los términos que se refieren a los pobres aparecen asociados a los sujetos que residen en la domus Dei, justamente, por encontrarse enfermos. Por ejemplo, en el Testimonio Anónimo, al que nos referimos en el punto anterior, de un peregrino que residió en el hospital en Jerusalén, se llamaba a los pacientes como "pobres enfermos»" ${ }^{22}$. Más interesante es el hecho de que el testimonio de este "Clérigo Anónimo» por momentos asocia el término pauperes al de aegri o al de infirmi para referirse indistintamente siempre a los mismos sujetos. Esto parecería demostrar que tampoco los vocablos latinos eran utilizados con un sentido estricto para designar las diferentes condiciones de los pacientes del hospital de Jerusalén. En efecto, estas palabras (pauperes, aegri o infirmi) eran utilizadas por el Clérigo Anónimo para referirse a residentes a los que se cuidaba y atendía de maneras específicas.

Por ejemplo, sobre quienes eran recibidos en el Hospital nos dice que «los pobres enfermos tienen la prioridad en el mencionado hospital, sea cual fuera la enfermedad que tengan ${ }^{23}$. Los «pobres enfermos» son designados en el original con los términos pauperes infirmi, mientras que en otro pasaje, en relación al servicio de búsqueda y transporte de los más débiles, bajo el rótulo de pauperum egrotancium:

Del mismo modo, si la naturaleza debilitada de los pobres enfermos fuera tal que no pudieran dirigirse al hospital de San Juan con el uso de su propia fuerza, misericordiosamente se los buscaría por la ciudad y serían transportados con humildad por los siervos del hospital ${ }^{24}$.

Como vemos, no se puede distinguir a partir de los términos latinos si el hospital atendía enfermos como los entenderíamos hoy. De la misma forma, podemos afirmar que tampoco es cierto que dichos términos guardasen sentidos exclusivos sobre quienes residían en el hospital. Si atendemos el primer pasaje citado, lo que observamos es que se daba una distinción entre pacientes según la condición específica, no en función de un término, sino por el sentido de la oración: la fuente señala que tienen prioridad los enfermos «sea cual sea la enfermedad que tengan». Así, destaca la tarea de cuidado y curación sobre otra que correspondería a la provisión de comida y hospedaje a peregrinos o hambrientos que podrían ser aquellos que no tuvieran «la prioridad en el mencionado hospital».

En relación con esto último, el segundo pasaje señala el servicio de búsqueda de los más débiles para ser ingresados y tratados en el hospital. Nuevamente, no se trataría aquí de la más «sencilla», aunque no necesariamente menos costosa, tarea de hospedar y alimentar gente. Del mismo modo, podemos asumir que los pacientes que necesitaban ser transportados tampoco serían simples peregrinos o humildes en busca de comida

22 Clérigo Anónimo, pp. 39, 52, 57 et passim.

23 «In primis igitur et primi dominacionis primatum in hospitali predicto optinent pauperes infirmi quacumque infirmitate detenti». Clérigo Anónimo, p. 39 (es nuestro el énfasis sobre pauperes infirmi).

24 «Item, si pauperum egrotancium natura virium fuerit defecta, ut proprii vigoris beneficio beati Iohannis hospitale non possint adire, misericorditer per villam queruntur et a famulis hospitalariis humiliter advehuntur». Clérigo Anónimo, pp. 39-40. 
y hospedaje. El hecho de que carecieran de movilidad propia nos hace pensar que su condición era grave, probablemente producto de heridas o enfermedades crónicas. Su atención, por lo tanto, podría no consistir simplemente en el alojamiento y cuidado, sino que era necesario que fuesen atendidos médicamente.

Por otro lado, con relación al segundo punto que indicamos más arriba sobre la aparición o no del término aegri (y sus flexiones correspondientes) en las fuentes anteriores a 1180, encontramos en otros registros, además de los estatutos y los documentos de la organización de la orden que figuran en el Cartulario General, que dicho término se registra en más de un pasaje. Por ejemplo, el Clérigo Anónimo lo utiliza al menos 20 veces en relación con los enfermos ${ }^{25}$, y seis para referirse a las enfermedades (egritudine $)^{26}$.

$\mathrm{Al}$ mismo tiempo, remitiéndonos a otras palabras utilizadas para designar a los enfermos, poseemos otros documentos de la orden que nos colocan nuevamente en la línea de señalar la dificultad de asignar un sentido tan estricto al vocablo aegri. En efecto, diversas fuentes en francés antiguo usan la palabra malade para referirse a los pacientes del hospital y su tratamiento a través de distintas terapias ${ }^{27}$. En la primera regla del Hospital, por ejemplo, aparece mencionada cinco veces ${ }^{28}$, mientras que en los estatutos de Roger des Moulins 20 veces $^{29}$.

\section{Los SERVICIOS MÉdicos del Hospital}

En torno al tercer punto que indicamos en la introducción, en función de las terapias llevadas a cabo en el hospital, observamos que se trataba de dispensar servicios orientados a la curación de los pacientes y no simplemente su cuidado. En efecto, poseemos toda una serie de testimonios de época que describen la tarea médica de los hospitalarios y su vocación por el pobre y el enfermo. Uno de estos es el del peregrino llamado Teodorico, quien visitó el Hospital en 1169 y lo describió de esta manera:

No le creería a nadie si no hubiese visto con mis propios ojos cuan espléndidamente está compuesto de salas con muchas habitaciones y literas y otras cosas que el pobre y el débil y el enfermo pueden usar. Cuan rico es este lugar y cuan excelentemente utiliza el dinero para el alivio de los pobres y cuan diligente es en la atención a mendigos. Transitando por

25 Clérigo Anónimo, pp. 39, 52, 57 et passim.

26 Clérigo Anónimo, pp. 45, 53, 55 et passim.

27 Ha sido señalado que un problema común entre los historiadores de los hospitalarios es el uso de la versión latina de la regla y de los estatutos de la orden en vez de la que figura en francés antiguo, cuya composición fue anterior y, por lo tanto, más fiel a los documentos originales. En efecto, su análisis, como indicó Luttrell, suele ser dejado de lado. Luttrell, Anthony T. "The Hospitallers' Early Written Records». En France, John y Zajac, William (eds.). The Crusades and their Sources. Essays Presented to Bernard Hamilton. London: Routledge, 1998, p. 153.

28 Cartulario General, n. ${ }^{\circ} 70$, pp. 63, 64, 67.

29 Cartulario General, n. ${ }^{\circ}$ 627, pp. 425, 426, 427 et passim. 
el edificio no pudimos de ningún modo juzgar el número de gente que reposa allí, pero vimos unas mil camas ${ }^{30}$.

Juan de Würzburgo se encontraba en Jerusalén por la misma época, cerca del año 1170. Sobre el Hospital de San Juan señaló que:

(...) en varias salas una gran multitud de gente enferma es reunida, algunas de ellas son mujeres y otros, hombres. Son atendidos y cada día alimentados con un gran gasto. El número total de personas al momento que yo estaba, supe por los sirvientes hablando de ello, era de dos mil personas enfermas. Entre la noche y el día a veces eran llevados afuera más de 50 cadáveres, pero una y otra vez nueva gente era admitida ${ }^{31}$.

Si hacemos caso de la descripción de los autores, el Hospital se dedicaba a la atención de enfermos y no simplemente de los hambrientos y los pobres, idea que se refuerza con la mención de Juan de Würzburgo de que un número significativo muere cada día. Más interesante resulta el hecho, como Piers Mitchell indica, que la fuente también menciona el gran gasto que implicaba la atención a los enfermos cada día. De hecho, la mención no es simplemente sobre las camas y la comida que la orden brindaba en su hospital, sino también sobre el gran costo que significaba la atención de los enfermos. En efecto, los hospitalarios utilizaban una enorme cantidad de recursos en la contratación de médicos y en las medicinas aplicadas con el propósito de atender y curar heridos y enfermos ${ }^{32}$. De la misma forma, se desplegaba toda una serie de servicios médicos que comprueban, una vez más, el propósito de curar a los pacientes y no simplemente cuidarlos.

En este sentido, conviene detenernos sobre una de las características más importantes del tipo particular de monacato que se desarrolló con las órdenes hospitalarias-militares que surgieron en Tierra Santa durante las cruzadas. Frente a la actitud contemplativa que se alejaba del saeculum en busca de Dios, propia del monacato tradicional, este nuevo tipo buscaba el acercamiento a Él a través de la lucha y, en el caso de los hospitalarios, a través del tratamiento de pobres y enfermos ${ }^{33}$.

30 «Que quantis edificiis decorata, quantis domiciliis et lectulis atque aliis utensiliis in usus pauperum et infirmorum atque debilium exhibendis habundans, quam in substantia pauperum recreationibus impendenda locuples, quam in ipsa egenorum sit sustentatione sollicita, nullus alteri verbis fidem posset facere nisi ipse propriis hoc oculis valeret deprehendere. Siquidem transeuntes per palatium numerum simul accumbentium nullo modo quivimus discernere, lectorum vero numerum millenarium vidimus excedere». HuYGENS, Robert (ed.). Peregrinationes tres: Saewulf, John of Wurzburg, Theodericus, Corpus Christianorum. Continuatio Medievalis, vol. 139. Turnhout: Brepols, 1994, pp. 157-158.

31 «(...) in quo per diversas mansiones maxima multitudo infirmorum tam mulierum quam virorum colligitur, fovetur et maxima expensa cotidie reficitur, quorum summam tunc temporis cum essem presens ab ipsis servitoribus hoc referentibus ad duo milla languentium fuisse cognovi, ex quibus aliquando infra noctem et diem plusquam quinquaginta mortui exportantur, iterum atque iterum pluribus de novo accedentibus». Huygens (ed.), Peregrinationes tres, pp. 131-132.

32 Mitchell, Piers D. Medicine in the Crusades, warfare, wounds and the medieval surgeon. Cambridge: Cambridge University Press, 2004, p. 65.

33 Riley-Smith, The Knights Hospitallers, pp. 69-70; Luttrell, Anthony T. "The Earliest Hospitallers». En Kedar, Benjamin; Riley-Smith, Jonathan y Hiestand, Rudolf (eds.). Montjoie: Studies in Crusade history in honor of Hans Eberhard Mayer. London: Variorum, 2007, p.41. 
Semejante tarea, asociada a la caridad monástica, constituía una novedad para el mundo occidental, aunque no para el de Oriente. En efecto, en el mundo bizantino la tradición monástica hospitalaria poseía larga data ${ }^{34}$. Asociado a su carácter médico caritativo, se ha sostenido que las instituciones orientales podrían haber influido o determinado el modelo hospitalario adoptado por la orden de San Juan ${ }^{35}$. Más allá de esta discusión, sobre la que no nos ocuparemos aquí, lo cierto es que el mismo espíritu de asistencia y atención médica de los monasterios bizantinos se expresaba en la primera regla de la orden, bajo el maestrazgo de Raimundo de Puy, y en toda una serie de documentos posteriores de la orden ${ }^{36}$. De la misma forma, otro maestro de la orden, Rogers de Moulins, convocaba en el 1182 a todos los comendadores de las diferentes casas del Hospital a servir al enfermo, ya que «por este beneficio merecerán tener parte en la gloria del cielo" ${ }^{37}$.

En este sentido, es posible entender que la orden del Hospital contratara médicos y otros profesionales para atender enfermos, lo que, insistimos, constituía un aspecto novedoso en la tradición monástica occidental, aunque no en la oriental. En efecto, el uso de dinero dentro del monasterio para la contratación de profesionales que atendieran a sujetos ajenos a la misma comunidad de hermanos era frecuente en el mundo bizantino ${ }^{38}$ y lo fue también para los hospitalarios en Jerusalén.

Los estatutos de la regla de Rogers des Moulins señalaban que para los enfermos del hospital de Jerusalén sean contratados cuatros médicos sabios ${ }^{39}$. De la misma forma, el documento de las «Regulaciones Administrativas» mencionaba que 1500 bezantes sean donados al hermano hospitalario a cargo del cuidado de los enfermos para contratar médicos y comida para los pacientes ${ }^{40}$.

De igual modo, en un pasaje más extenso, el Clérigo Anónimo señalaba lo siguiente:

34 Ya desde el siglo IV existieron diferentes tipos de instituciones hospitalarias a lo largo y ancho del imperio que, sostenidas por dignidades de la iglesia griega o miembros de la aristocracia o la realeza, desplegaban toda una serie de tareas hospitalarias desde los diversos monasterios del mundo bizantino. Miller, The birth of the Byzantine hospital.

35 TouAt, François-Olivier. «La Terre Sainte: un laboratoire hospitalier au Moyen Âge?». En Bulst, Neithard y SpIEss, Karl (eds.). Sozialgeschichte Mittelalterlicher Hospitäler. Ostfildern: Jan Thorbecke Verlag, 2007, pp. 169-211; Amouroux, Monique. "Colonization and creation of hospitals: the eastern extension of western hospitality in the eleventh and twelfth centuries». Mediterranean Historical Review, 1999, vol. 14, pp. 31-43.

36 Cartulario General, n. ${ }^{\circ} 70$, pp. 62-68.

37 Cartulario General, n. ${ }^{\circ} 627$, p. 426.

38 Miller, «Byzantine hospitals».

39 Cartulario General, n. ${ }^{\circ}$ 627, p. 426.

40 Este documento se ubica en el Codex Vat. Lat. 4852, que contiene numerosos documentos importantes de los Hospitalarios. La mayoría de ellos fueron integrados en la obra de Delaville le Roulx. Sin embargo, llamativamente los folios entre el 83r y el $104 \mathrm{r}$ no fueron tenidos en cuenta por este autor. Su edición fue realizada por Susan Edgintong: Edgington, Susan. «Administrative Regulations for the Hospital of St John in Jerusalem dating from the 1180s». Crusades, 2005, vol. 4, pp. 21-37. (En adelante se citará como Regulaciones Administrativas y el número de página). Sobre la composición del documento original, la fecha y los criterios de edición véase la introducción de la autora al artículo. 
Pero como [los hermanos], que desconocen la física inferior, pueden presentar ciegamente una combinación de muchos [alimentos] a los que comen, el santo convento del hospital encomendó con santidad y providencia sus enfermos a la pericia de los teóricos, al fiel cuidado de los [médicos] prácticos. (...) Así pues, como debe evitarse el peligro de la mala fama, en verdad hay en el hospital cuatro médicos doctos en medicina. Son estipendiarios de la casa, para que no asuman una preocupación diferente a los enfermos del hospital. Ellos también son obligados por un juramento, del que no deben ser recordados ni disuadidos. Estos pocos -que esperan fuera del hospital hasta su hora- saben qué cosas son necesarias para la salud de sus enfermos, ya sea a través de electuarios o de otras medicinas. En efecto, los médicos no proporcionan ninguna de sus medicinas propias a los enfermos, sino todas aquellas que sean suministradas por la casa ${ }^{41}$.

En función de esta descripción observamos que, en primer lugar, al igual que en los estatutos de Roger de Moulins, eran contratados cuatro médicos «sabios», es decir, aquellos que prescribían terapias e indicaban a los "prácticos» qué debían realizar. En segundo lugar, que existe una distinción entre los «conocedores de la física inferior» y los otros, «encargados del fiel cuidado». De la misma forma, en una carta de 1184 dirigida a Roger de Moulins, el papa Lucio III, en relación con la tarea médica del Hospital, mencionaba la presencia de cuatro medici y, también, el mismo número de cyrurgici trabajando en el Hospital, lo que permite entender la existencia de una distinción jerárquica en la organización de las tareas de la institución ${ }^{42}$. De este modo, si atendemos a lo señalado al comienzo sobre el grado de «medicalización» de las instituciones hospitalarias medievales como herramienta analítica para definir el grado de desarrollo y alcance del hospital en Jerusalén, queda claro, a partir de la evidencia documental, que la institución hierosolimitana era sin duda un hospital que contaba con presencia regular de médicos que actuaban para la curación de los pacientes.

En el mismo sentido, por otro lado, además de señalarse la contratación de profesionales, el Clérigo Anónimo agregaba que eran provistos de toda la medicina necesaria, sin tener que aplicar ninguna propia para la atención de los pacientes. Al igual que en las instituciones orientales (bizantinas y árabes) a las que nos referimos al comienzo, tanto los profesionales como los suministros necesarios para la curación de los enfermos derivaban del estipendio de la casa.

Las fuentes del Hospital también nos brindan información sobre la contratación de otros asistentes de menor rango y sirvientes designados para distintas tareas. El Clérigo Anónimo señala la existencia de cirujanos y minutores a cargo de sangrar a los pacientes ${ }^{43}$.

41 «Sed quoniam inferioris phisice prorsus ignari nonnisi ceco casu plura degustantibus huismodi possunt exhibere temperamentum, sanctus hospitalis conventus theoricorum peritie fideli practicorum cure infirmos suos sancte commisit ac provide. (...) Propter ergo cum infame periculum evitandum, sunt enim in hospitali quatuor medici phisicam docti, ita domus stipendiarii, ut aliquam curam infirmis hospitalis alienam non presumant, qui et iuramento constringuntur quod nullius admonicione, plura nullius dissuasione, pauci -hora exspectent ab hospitali- queque ad salutem infirmorum suorum noverint necessaria, hinc in electuariis, inde in aliis medicinis, quia de suo nulla egris medici subpeditant medicamenta, sed de domo illis ministrantur omnia». Clérigo Anónimo, pp. 45-46.

42 Cartulario General, n. ${ }^{\circ}$ 690, p. 458.

43 Clérigo Anónimo, pp. 21-22 / 46-47 
En los estatutos de Rogers des Moulins se menciona la existencia de nueve sargentos de la orden asignados a cada sala para lavar los pies, cambiar las sábanas, alimentar a los pobres y estar al servicio de los enfermos ${ }^{44}$ para todo lo que necesitaran ${ }^{45}$.

Por otro lado, sabemos que existían en el hospital de Jerusalén diferentes salas que podrían haber servido, como en algunos hospitales bizantinos y árabes ${ }^{46}$, para la atención de los pacientes según su grado de convalecencia o su sexo. El Clérigo Anónimo mencionaba que eran once. Señalaba además, al igual que Juan de Würzburgo, que existían algunas especiales para las mujeres ${ }^{47}$, factor que corrobora que personas de ambos sexos eran tratadas en el hospital ${ }^{48}$. En efecto, sabemos que muchas de ellas eran mujeres parturientas que se dirigían a la domus Dei para dar a luz bajo el cuidado de las hermanas hospitalarias. En relación con este último punto conocemos otra de las tareas de asistencia que la orden del Hospital desplegó en Jerusalén: la adopción y el cuidado de niños huérfanos y mujeres embarazadas, aspecto que analizaremos en una sección posterior.

Por otro lado, conviene aclarar que la asignación de ciertos tipos de comidas, como los que se distribuían entre los pacientes del Hospital, también implicaba un gasto enorme. La terapia médica medieval más importante se vinculaba a la prescripción de alimentos para restaurar el equilibrio de los humores del paciente ${ }^{49}$. En más de un documento del Hospital se instruía a tratar a los enfermos con comidas de diferente tipo, según la condición de cada uno, y las formas correctas de administrarlas ${ }^{50}$. En este sentido, las fuentes de la orden describen la existencia de una cocina especial para los enfermos dentro del complejo hospitalario donde se preparaban las comidas para cada paciente según las indicaciones de los médicos ${ }^{51}$.

En los estatuos de 1176, por ejemplo, se establecía la entrega de pan blanco a los enfermos de la domus $D e i^{52}$. Semejante privilegio no era común en las instituciones hospitalarias del medioevo. En este punto, los estatutos de Rogers de Moulins también evidencian la cantidad de recursos dispuestos para el tratamiento del paciente. Cada priorato de la orden debía enviar al convento central en Jerusalén determinada cantidad de bienes designados para el hospital. Así, cada una de las casas, tanto en Oriente y en Europa, era requerida con distintos elementos que iban desde fustanes y telas para las camas de los enfermos hasta la solicitud de azúcar para la elaboración de jarabes y otras medicinas ${ }^{53}$.

44 Cartulario General, n. ${ }^{\circ} 627$, pp. 425-429.

45 Regulaciones Administrativas, pp. 24-26.

46 HamarneH, «Development of hospitals in Islam»; Miller, «Byzantine hospitals».

47 Clérigo Anónimo, p. 41.

48 Juan de Würzbugo, p. 131.

49 En efecto, la enfermedad era entendida como un desbalance de los humores que componían el cuerpo humano. Por lo tanto, para recuperar el estado de salud era preciso restaurar el equilibrio humoral. Las terapias más adecuadas, en función de la teoría médica galénica-hipocrática, consistían principalmente en asignar alimentos específicos, así como la realización de ciertos ejercicios. NutTon, Vivian. "Medicine in the Greek World, 800-50 BC». En Conrad, Lawrence et al. (eds.). The Western Medical Tradition. 800 BC to $A D$ 1800. Cambridge: Cambridge University Press, 1995, pp. 26-27.

50 Regulaciones Administrativas, pp. 32, 34; Clérigo Anónimo, p. 44.

51 Clérigo Anónimo, p. 43.

52 Cartulario General, n. ${ }^{\circ} 494$, pp. 339-340.

53 «Cet si comanda, quand le conseil fu tenus des freres, sur ce que le prior de l'Ospital de France mandast chascun an en Jerusalem $\mathrm{C}$ dras de coton tainz por renoveler les covertours des povres, et les contast en sa 
De la misma forma, las Regulaciones Administrativas ponen en evidencia el importante despliegue y cuidado en la atención de los enfermos. Se señala además la existencia de médicos que en cada sala deben observar a los pacientes y registrar su condición para prescribir qué medicinas deben ser aplicadas:

Fue ordenado por el maestro del hospital y del capítulo general que cada día cada enfermo tenga medio pan blando y pan suficiente de la casa, y el mismo vino como en el convento. Los médicos deben observar atentamente la condición de los enfermos y qué enfermedades ellos padecen, y deben observar las orinas y entregar los jarabes y los electuarios y las otras cosas que son necesarias para los enfermos, y separar las cosas contrarias y entregar las beneficiosas, y tanto como ellos [los médicos] vean más enfermos y más débiles, tanto más sean atendidos para procurar su salud ${ }^{54}$.

Nuevamente, nada en este pasaje permite señalar que los tratamientos a los enfermos del Hospital no estuviesen orientados a su curación. Por otro lado, otra sección del relato del Clérigo Anónimo hace referencia a la elaboración de remedios a partir del uso de hierbas y piedras:

¡Oh! ¡Qué santa casa, que conoce cuántas beatas virtudes hay en las piedras, cuántas fuerzas hay dentro de las hierbas puestas misericordiosamente por el Creador, de modo que el hombre en su exilio pudo remediar con ellas los daños de su naturaleza corruptible, a causa del pecado del primer padre! $!^{55}$

En este pasaje el autor da cuenta del uso de plantas que poseían propiedades farmacológicas útiles para «remediar los daños de la naturaleza corruptible del hombre». En el mismo sentido, se refiere al uso de piedras, lo que se vinculaba a la creencia de que algunas poseían diferentes cualidades humorales y podían ser utilizadas para revertir el exceso de algún humor en el paciente (litoterapia). Este hecho refuerza nuestra afirmación de

responsion aveuc ceaus qui seront donez à la maison en son prioré en aumone. Eu icelle meisme maniere et à cel conte le prior de l'Ospital de S. Gile autretant de dras de coton achate chascun an, et mande en Jerusalem avec ceaus qui seront donez en son prioré por l'amor de Deu as povres de l'Ospital. Le prior d'Ytalie chascun an mande en Jerusalem as seignors povres II M aunes de fustaines de diverses colors, que il conte chascun an en sa responsion. Et le prior de Pise mande autresi auterant de fustaines. Et le prior de Veneise autressi, et tout soit conté sur lor responsion. Et le bailliz autressi de desa mer soient veillant à cest meisme servise. Dont le bailli d'Antioche mande en Jerusalem II M canes de toile coton as covertors de malades. Le prior de Monpelerin mande en Jerusalem II quintaus de sucre por le syrop, et les medecines, et les laitures des malades. Au cel meisme servise le bailli de Tabarie en mande autretant. Le prior de Constantinople mande por les malades CC feutres». Cartulario General, n. ${ }^{\circ}$ 627, pp. 426-427.

54 «Ordene fu dou maistre de lospital et dou general chapistre que chascun jor chascun malade ait demi pain mouflet et dou pain de la maison assez, et tel vin com le covent. Les mieges d[o]ivent regarder ententivement les qualitez des malades et quelz maladies ils ont, et doivent regarder les orines et doner les syrops et les laituaires et les autres $/ 89 \mathrm{v} /$ choses qui sont necessaires as malades et desfendre les choses contraires et doner les profitables, et de tant comil les verront plus malades et plus febles, tant soient plus apareillielliez encor lor sante procurer». Regulaciones Administrativas, p. 24.

55 «O quam beata domus, quod beate considerans lapidibus virtutes, herbis vires a creatore misericorditer insitas, ut scilicet homo per eas a primo primi parentis excessu corruptibilis nature incommodis in hoc suo possit mederi exilio». Clérigo Anónimo, pp. 47-48. 
que lo que se buscaba en el hospital en Jerusalén era curar al paciente y no simplemente cuidarlo. El problema en este punto reside en las interpretaciones que anacrónicamente asocian estos tratamientos a procedimientos rudimentarios y sostienen la distinción entre cuidar y curar como constitutiva de un carácter diferenciador entre las instituciones ${ }^{56}$.

\section{$4 \quad$ El CUIDADO A LOS NIÑOS}

Este aspecto de la orden, si bien ha sido mencionado en más de una ocasión dentro de la lista de actividades caritativas que los hospitalarios desplegaron en Jerusalén, no fue tenido en cuenta por ningún estudio como tema específico. La razón de esta ausencia podría derivar del hecho de que no existe tampoco ningún trabajo acerca de la historia de la infancia en el Reino Latino de Jerusalén ${ }^{57}$.

Sabemos a partir de diferentes documentos que el hospital de San Juan funcionaba en la Ciudad Santa también como orfanato y que esta tarea le otorgaba a la orden un importante reconocimiento en el mundo del Mediterráneo oriental y en el mundo europeo $^{58}$. Efectivamente, más de un cronista ha destacado el valor y las bondades de dicha tarea. En un testimonio anónimo anglonormando eran descritos los cuidados brindados a los niños y a las mujeres encintas dentro del Hospital ${ }^{59}$ :

Escuchen de aquellos [niños] que son encontrados / por la gracia de Dios ellos son muchos / de los que no sabemos quienes los han engendrado / ni quienes los han concebido ni abandonado / todos son bondadosamente recibidos / del mismo modo son alimentados/ sean varones o mujeres $(. . .)^{60}$.

Estos aspectos se corroboran en otra serie de documentos previos de la orden. Nuevamente, el Clérigo Anónimo mencionaba que el Hospital disponía que los bebés que fueran abandonados en la domus Dei, fueran llevados al interior del palacio donde eran

56 Véase Edgington, «The Hospital of St John in Jerusalem», p. xxi.

57 Edgington, Susan. «The First Crusade: Expanding the Historiography». En Edgington, Susan y García-Guijarro, Luis (eds.). Jerusalem the Golden. The Origins and Impact of the First Crusade. Turnhout: Brepols Publishers, 2014, pp. 1-7. En cambio, la historia de la infancia en Europa occidental durante la Edad Media posee una larga tradición de trabajos. Desde la obra de Phillipe Ariès en 1960, hasta producciones más recientes, aparecieron numerosos trabajos que buscaron entender cómo las sociedades del mundo medieval conceptualizaban la niñez y cuál era el lugar de los más pequeños en la sociedad. ArıÈs, Philippe. L'enfant et la vie familiale sous l'Ancien Régime. Paris: Éditions du Seuil, 1973; cfr. Shahar, Shulamith. Childhood in the Middle Ages. London: Routledge, 1990; Orme, Nicholas. Medieval Children. London: Yale University Press, 2001; Cunningham, Hugh. The invention of Childhood. London: BBC Books, 2006.

58 Para la atención a la infancia y las instituciones que funcionaron como orfanatos en el mundo oriental medieval, véase una introducción al tema en Miller, Timothy. The Orphans of Byzantium. Child Welfare in the Christian Empire. Washington D. C.: The Catholic University of America Press, 2003.

59 Beltjens, Alain. «Un commentaire anglo-normand d'une partie importante de la Règle et de quelques fragments de réglementations diverses de l'hôpital de Saint-Jean de Jérusalem». Société de l'Histoire et du Patrimoine de l'Ordre de Malte. Numéro spécial, 2006, vol. 16, pp. 1-123.

60 «Oez de ceus ke sunt trovez / Dunt, merci Deu, sunt acez / Ke l'en ne seit ki engendrat / Ne kes conceut ne ke las getat. / Tut i sunt beau recuilli, / Ensement i sunt nurri, / Seient males .u. femeles». BeLTJENS, «Un commentaire anglo-normand», p. 65. 
colocados junto a los recién nacidos de las madres residentes en el hospital en cunas hechas especialmente para ellos:

Pero ciertamente las madres con sus rostros cubiertos abandonan a escondidas allí a los nińos, a causa de la misericordia -ya conocida por muchos- de aquella casa. Si alguna hubiera parido gemelos, habiendo conservado uno, al otro lo entregaba para que fuera alimentado por el beato Juan, que no se opone a esto; de esta manera, ninguno queda abandonado ${ }^{61}$.

Esta imagen coincide con lo mencionado en los estatutos de Rogers des Moulins, donde era establecido que:

(...) pequeñas cunas sean hechas para los bebés de las mujeres peregrinas que nacen en la casa, de modo que la criatura permanezca en una parte sola, y que el bebé lactante no esté en ningún peligro por el movimiento de la madre ${ }^{62}$.

Por lo tanto, además de recibir y poner bajo su protección a los nińos abandonados, los hermanos de San Juan ingresaban a las mujeres embarazadas para que dieran a luz dentro del hospital. El Clérigo Anónimo agregaba que a esta tarea se sumaba la alimentación de los bebés lactantes abandonados en las puertas de la domus Dei. Para ello el Hospital disponía de nodrizas designadas por las hermanas del convento, encargadas también de la alimentación y cuidado de las madres que residieran en la casa:

No obstante, si una madre de uno solo [de los niños] no alcanza a alimentarlo por circunstancias desfavorables, ella hace saber su decisión al maestro de la casa. Por lo tanto, si la enfermedad fuera la causa [del abandono], ese hombre piadoso designará al niño otra nodriza para su fiel y continua custodia. Si verdaderamente la pobreza hubiera sido la causa contra la alimentación del niño, el maestro la presenta a ella y al niño a la nodriza y, al instante, algo [de dinero] es llevado por él a modo de don, de beneficio para su consuelo. Así pues, las nodrizas de tales niños abandonados, cada uno de ellos hijo adoptivo del beato Juan, que podrían ser incluso mil, reciben doce talentos por año y en toda solemnidad son provistas de nueve raciones de alimento de la casa, del mismo modo que los hermanos mismos, tanto en cantidades de porciones como en las variedades de platos ${ }^{63}$.

${ }_{61}$ «Si vero puerpere aut inedia desolate aut insolito nature cursu materne pietatis oblite, parvulos suos abiecerint, a primis inventoribus ad hospitale deportati humillime suscipiuntur, nutricibus illico quesitis lactis alimonia educandi committuntur, sed robustiori cibo confortandi in ipsa domo enutriuntur. Sed vero matres clanculo fronte obducta infantes illic abiciunt, immo a multis iam cognita domus illius misericordia, si qua gemellos fuerit enixa, uno retento reliquum beato Iohanni nutriendum nullo rebellante palam relinquo». Clérigo Anónimo, p. 55.

62 «Cet si establi que petiz bers fucent fait por les enfans des femes pelerines qui naissent en la maison, si que il gisent à une part soul, et que li enfant alaitant n'en aient aucun ennui par la mesaise de lor mere». Cartulario General, n. ${ }^{\circ} 627$, p. 426.

${ }^{63}$ "Attamen si tantum et unius mater illum quocumque circumstante incomodo non sufficiat enutriente, summo magistro domus suum propalat decidium. Si ergo egritudo fuerit in causa, continuo pius vir ille puerum fideli alterius nutricis custodie deputat. Si vero paupertas nutriendo infantulo [fuerit in causa, convenit magister cum illa tamquam de alio cum aliena, confestim insuper sibi aliquo collato consolatorii emolumenti beneficio. Nutrices itaque talium abiectorum scilicet filiorum beati Ihoannis adoptorum singule, licet essent mille, XII. habent talenta per annum et in omni sollempnitate. IX. lectionum de cibariis domus procurantur, sicut et ipsi fratres tam in porcionum quantitatibus quam in ferculorum varietatibus». Clérigo Anónimo, p. 55. 
Este último aspecto de las tareas desplegadas por los hospitalarios refuerza el argumento hasta aquí desarrollado. La atención de bebés y madres, el cuidado de niños huérfanos y el empleo de nodrizas, son otras de las tareas que implicaron la gran cantidad de recursos dispuestos por el Hospital para el tratamiento de los necesitados.

\section{Conclusiones}

Por lo visto hasta este punto es posible sostener que el gran hospital de Jerusalén antes de 1180 ya brindaba cuidado y atención médica a enfermos con el propósito de su curación. La descripción del Clérigo Anónimo, y otros documentos que datan de estos años, describen prácticas ya existentes en la institución para esta época, lo que permite corroborar el despliegue médico dispensado por la orden de San Juan.

Los problemas de interpretar el tipo de atención de los sujetos que residían en la institución como factor decisivo para definir su grado de desarrollo parten de disminuir la importancia terapéutica desplegada en esta institución bajo criterios que muchas veces son anacrónicos. Vimos cómo, por ejemplo, en el mundo medieval la distinción entre pauper y aegri no poseía el significado que actualmente podríamos atribuirle. De hecho, también observamos que ambos podían aparecer asociados o ser usados con el mismo significado indistintamente.

Al mismo tiempo, señalamos algunas de las terapias y la magnitud de los recursos que el Hospital disponía y empleaba -descritos en los estatutos y otros documentos- para las tareas de asistencia y atención al enfermo, en una diversidad de actividades que nos permiten afirmar, nuevamente, que el Hospital no se dedicaba simplemente a cuidar, sino que también curaba a los residentes que lo necesitaran.

Por último, mencionamos al comienzo que el debate sobre la «medicalización» de las instituciones hospitalarias medievales ha contribuido a reforzar las distinciones entre instituciones modernas y premodernas. En este sentido, nos preguntamos si este tipo de lecturas no condicionaría la interpretación que se realizó sobre las instituciones hospitalarias del pasado toda vez que analizadas en términos actuales la disposición de profesionales sería casi necesariamente vista como rudimentaria. En este punto, resulta interesante que el hospital en Jerusalén, como algunos de los más importantes del mundo árabe o bizantino de la época, contrataba una gran cantidad de profesionales con especialidades diversas, factor que refuerza la afirmación de que se trató de una institución cuyo despliegue resultaba sumamente significativo en los términos propios de la práctica médica medieval.

\section{REFERENCIAS BIBLIOGRÁFICAS}

Allan, Nigel. «Hospice to Hospital in the Near East: An Instance of Continuity and Change in Late Antiquity». Bulletin of the History of Medicine, 1990, vol. 64, n. ${ }^{\circ} 3$, pp. 446-462.

Amouroux, Monique. "Colonization and creation of hospitals: the eastern extension of western hospitality in the eleventh and twelfth centuries». Mediterranean Historical Review, 1999, vol. 14, pp. 31-43. 
Ariès, Philippe. L'enfant et la vie familiale sous l'Ancien Régime. Paris: Éditions du Seuil, 1973.

Beltjens, Alains. «Le récit d'une journée au Grand Hôpital de Saint-Jean de Jérusalem sous le règne des derniers rois latins ayant résidé à Jérusalem ou le témoignage d'un clerc anonyme conservé dans le manuscrit Clm. 4620 de Munich». Société de l'Histoire et du Patrimoine de l'Ordre de Malte. Numéro spécial, 2004, vol. 14, pp. 1-79.

Beltjens, Alain. "Un commentaire anglo-normand d'une partie importante de la Règle et de quelques fragments de réglementations diverses de l'hôpital de Saint-Jean de Jérusalem». Société de l'Histoire et du Patrimoine de l'Ordre de Malte. Numéro spécial, 2006, vol. 16, pp. $1-123$.

Boas, Adrian. Archeology of the Military Orders. London: Routledge, 2006.

Brodman, James W. «Religion and Discipline in the Hospitals of Thirtennth-century France». En Bowers, Barbara S. (ed.). The Medieval Hospital and Medical Practice. London-New York: Routledge, 2007, pp. 123-132.

Brodman, James. Charity and Welfare: Hospitals and the Poor in Medieval Catalonia. Philadelphia: University of Pennsylvania Press, 1998.

BronsteIn, Judith. "Servus pauperum Christi: los servicios de los Hospitalarios a los peregrinos en el Oriente Latino, siglos XI-XIII". Memoria y Civilización, 2013, vol. 16, pp. 219-236.

Congourdeau, Marie-Hélène. "La médecine byzantine. Une réévaluation nécessaire». Revue du Praticien, 2004, vol. 54, n. ${ }^{\circ}$ 15, pp. 1733-1737.

Constantelos, Demetrios J. Byzantine philanthropy and social welfare. New Brunswick-New Jersey: Rutgers University Press, 1968.

Cunningham, Hugh. The invention of Childhood. London: BBC Books, 2006.

Davis, Adam J. The Medieval Economy of Salvation. Charity, Commerce, and the Rise of the Hospital. Ithaca: Cornell University Press.

Delaville le RoulX, Joseph (ed.). Cartulaire général de l'Ordre des Hospitaliers de S. Jean de Jerusalem, 4 vols. Paris: Académie Royale des Inscriptions et Belle-Lettres, 1895-1906.

Demurger, Alain. Les Hospitaliers. De Jerusalem à Rhodes. 1050-1317. Paris: Éditions Tallandier, 2015.

Dous, Michael. «The origins of the Islamic hospital: myth and reality». The Bulletin of the History of Medicine, 1987, vol. 61, pp. 367-390.

Edgington, Susan. «Administrative Regulations for the Hospital of St John in Jerusalem dating from the 1180s». Crusades, 2005, vol. 4, pp. 21-37.

Edgington, Susan. "The First Crusade: Expanding the Historiography». En Edgington, Susan y García-Guijarro, Luis (eds.). Jerusalem the Golden. The Origins and Impact of the First Crusade. Turnhout: Brepols Publishers, 2014, pp. 1-7.

Edgington, Susan. "The Hospital of St John in Jerusalem». En Amar, Zohar; Lev, Efrain y Schwartz, Joshua (eds.). Medicine in Jerusalem throughout the ages. Tel Aviv: Eretz, 1999, pp. XXI-XXII.

Hamarneh, Sami. «Development of hospitals in Islam». Journal of the History of Medicine and Allied Sciences, 1962, vol. 17, pp. 366-384.

Horden, Peregrine. «How Medicalised Were Byzantine Hospitals?». Medicina \& Storia, 2006, vol. 5 , n. ${ }^{\circ} 10$, pp. $45-74$.

Horden, Peregrine. «The Earliest Hospitals in Byzantium, Western Europe, and Islam». The Journal of Interdisciplinary History, 2005, vol. 35, n. ${ }^{\circ} 3$, pp. 361-389.

Huygens, Robert (ed.). Guillaume du Tyr. Chronicon, Corpus Christianorum. Continuatio Medievalis, vols. 63, 63A. Turnhout: Brepols Publishers, 1986. 
Huygens, Robert (ed.). Peregrinationes tres: Saewulf, John of Wurzburg, Theodericus, Corpus Christianorum. Continuatio Medievalis, vol. 139. Turnhout: Brepols, 1994.

Kedar, Benajmin. "A note on Jerusalem's Bimaristan and Jerusalem's Hospital». En Borchardt, Karl; Jaspert, Nikolas y Nicholson, Helen (eds.). The Hospitallers, the Mediterranean and Europe Festschrift for Anthony Luttrell. London: Ashgate, 2007, pp. 7-11.

Kedar, Benjamin. "A twelfth-century description of the Jerusalem Hospital». En Nicholson, Helen (ed.). The Military Orders: fighting for the faith and caring for the sick. Vol. 2. London: Ashgate, 1998.

Luttrell, Anthony T. «The Earliest Hospitallers». En Kedar, Benjamin; Riley-Smith, Jonathan y Hiestand, Rudolf (eds.). Montjoie: Studies in Crusade history in honor of Hans Eberhard Mayer. London: Variorum, 2007.

Luttrell, Anthony T. «The Hospitallers' Early Written Records». En France, John y Zajac, William (eds.). The Crusades and their Sources. Essays Presented to Bernard Hamilton. Londres: Routledge, 1998, pp. 135-154.

Miller, Timothy. «Byzantine hospitals». Dumbarton Oaks Papers, 1984, vol. 38, pp. 53-63.

Miller, Timothy. The birth of the hospital in the Byzantine Empire. Baltimore-London: The John Hopkins University Press, 1997.

Miller, Timothy. The Orphans of Byzantium. Child Welfare in the Christian Empire. Washington D. C.: The Catholic University of America Press, 2003.

Mitchell, Piers D. Medicine in the Crusades, warfare, wounds and the medieval surgeon. Cambridge: Cambridge University Press, 2004.

Nutton, Vivian. "Medicine in the Greek World, 800-50 BC». En Conrad, Lawrence et al. (eds.). The Western Medical Tradition. 800 BC to AD 1800. Cambridge: Cambridge University Press, 1995, pp. 26-27.

Orme, Nicholas. Medieval Children. London: Yale University Press, 2001.

Pringle, Denys. The Churches of the Crusader Kingdom of Jerusalem; A Corpus 3: The City of Jerusalem. Cambridge: Cambridge University Press, 1993.

Ragab, Ahmed. The Medieval Islamic Hospital. Medicine, Religion and Charity. New York: Cambridge University Press, 2015.

Riley-Smith, Jonathan. The Knights Hospitallers in the Levant, c. 1070-1309. Hampshire: Palgrave Macmillan, 2012.

Risse, Gunter. Mending Bodies, saving souls: A history of hospitals. New York: Oxford University Press, 1999.

ShaHAR, Shulamith. Childhood in the Middle Ages. London: Routledge, 1990.

TouAti, François-Olivier. «La Terre Sainte: un laboratoire hospitalier au Moyen Âge?». En BulsT, Neithard y Spiess, Karl (eds.). Sozialgeschichte Mittelalterlicher Hospitäler. Ostfildern: Jan Thorbecke Verlag, 2007, pp. 169-211. 